\title{
Knowledge and perception towards net care and repair practice in Ethiopia
}

\author{
Ayele Zewde ${ }^{1}$, Seth Irish², Adugna Woyessa ${ }^{3}$, Yonas Wuletaw ${ }^{3}$, Honelgn Nahusenay ${ }^{1 *}$ (D), Semira Abdelmenan ${ }^{1}$, \\ Meaza Demissie ${ }^{1}$, Hanna Gulema' ${ }^{1}$ Gunawardena Dissanayake ${ }^{4}$, Sheleme Chibsa ${ }^{4}$, Hiwot Solomon ${ }^{5}$, \\ Meseret A. Yenehun ${ }^{5}$, Amha Kebede 3 , Lena M. Lorenz ${ }^{6}$, Gabriel Ponce-de-Leon', Joseph Keating ${ }^{8}$, \\ Alemayehu Worku' and Yemane Berhane ${ }^{1}$
}

\begin{abstract}
Background: Long-lasting insecticidal nets (LLINS) are a key malaria control intervention. Although LLINs are presumed to be effective for 3 years under field or programmatic conditions, net care and repair approaches by users influence the physical and chemical durability. Understanding how knowledge, perception and practices influence net care and repair practices could guide the development of targeted behavioural change communication interventions related to net care and repair in Ethiopia and elsewhere.

Methods: This population-based, household survey was conducted in four regions of Ethiopia [Amhara, Oromia, Tigray, Southern Nations Nationalities Peoples Region (SNNPR)] in June 2015. A total of 1839 households were selected using multi-stage sampling procedures. The household respondents were the heads of households. A questionnaire was administered and the data were captured electronically. STATA software version 12 was used to analyse the data. Survey commands were used to account for the multi-stage sampling approach. Household descriptive statistics related to characteristics and levels of knowledge and perception on net care and repair are presented. Ordinal logistic regression was used to identify factors associated with net care and repair perceptions.

Results: Less than a quarter of the respondents (22.3\%: 95\% Cl 20.4-24.3\%) reported adequate knowledge of net care and repair; $24.6 \%$ (95\% Cl 22.7-26.5\%) of the respondents reported receiving information on net care and repair in the previous 6 months. Thirty-five per cent of the respondents (35.1\%: 95\% Cl 32.9-37.4\%) reported positive perceptions towards net care and repair. Respondents with adequate knowledge on net care and repair (AOR 1.58: 95\% $\mathrm{Cl} 1.2-2.02$ ), and those who discussed net care and repair with their family (AOR 1.47: 95\% Cl 1.14-1.89) had higher odds of having positive perceptions towards net care and repair.

Conclusions: The low level of reported knowledge on net care and repair, as well as the low level of reported positive perception towards net repair need to be addressed. Targeted behavioural change communication campaigns could be used to target specific groups; increased net care and repair would lead to longer lasting nets.
\end{abstract}

Keywords: Net care and repair, Knowledge, Perception, LLIN, Ethiopia

\section{Background}

Malaria continues to be one of the biggest public health problems in Ethiopia; 75\% of the country's land mass is considered to be malarious and $60 \%$ of the population

\footnotetext{
*Correspondence: h.nahu2000@gmail.com;

honelgnnahusenay@addiscontinental.edu.et

${ }^{1}$ Addis Continental Institute of Public Health, Addis Ababa, Ethiopia

Full list of author information is available at the end of the article
}

resides in these areas [1, 2]. According to the 2011 Malaria Indicator Survey (MIS), around 77\% of cases were due to Plasmodium falciparum and the remainder was Plasmodium vivax [3]. The long-lasting insecticidal net (LLIN) is a key malaria control intervention, and is currently a priority for scale-up in areas where malaria transmission occurs. If properly used, LLINs act not only as a physical barrier against biting mosquitoes, but also substantially reduces malaria transmission [4-7]. 
As in most countries in sub-Saharan Africa, LLINs are an important tool for malaria prevention and control in Ethiopia [8]. According to the 2011 MIS, 55\% of households reported owning at least one LLIN [3], with varying levels of net ownership across regions in Ethiopia [9, 10]. The level of LLIN utilization also showed variation by region in Ethiopia [3]. According to the World Health Organization Pesticide Evaluation Scheme (WHOPES), LLINs are expected to remain effective for 3 years under field conditions [11]. However, varying levels of durability in the field have been reported [12-14], with only a few studies confirming their usefulness over the entire 3 years $[15,16]$.

The duration of physical integrity and chemical effectiveness of LLINs are often influenced by factors such as household condition, frequency of washing, type of cooking fuel used, the location of the kitchen inside the house, net repair practice $[17,18]$, and the level of care given to nets in general [19-21]. Increased net care and repair practices could increase the longevity of the net: keeping the net away from children, pests and rodents; rolling up the net when not in use; and washing the net gently were some of the recommended net care approaches [19-21]. In addition, it is also recommended to repair any small hole in the net immediately [22]. Repairing small holes immediately could prolong the physical durability of the LLIN, although hole repair practices are uncommon [23, 24].

For households to employ net care and repair practices, knowing how to adequately care for and repair a net is the starting point. Given the variation in what is reported as net care and repair [21, 22, 25], it is important that knowledge is assessed contextually. Perception towards net care and repair practice (e.g., holes can be fixed, fixing holes will make the net last longer, others in the community are also fixing holes, and confidence in one's ability to repair a net) is also important, as behavioural change processes are influenced by perception [21].

Although, many studies have systematically assessed issues related to LLIN longevity, there is little evidence on knowledge and perception towards net care and repair in Ethiopia. The purpose of this study is to measure the level of knowledge about net care and repair approaches, identify perceptions towards net care and repair, and isolate factors that influence these perceptions among households in Ethiopia.

\section{Methods}

\section{Study site}

This study was conducted in four regional states of Ethiopia: Amhara, Oromia, Southern Nations Nationalities Peoples Region (SNNPR), and Tigray. About 86\% of the population of Ethiopia inhabit these regions [26].
The overall prevalence of malaria in the study site was between 0.7 and 1.3\% in areas below $2000 \mathrm{~m}$; SNNPR had the highest prevalence (2.5\%) followed by Amhara (2.0\%), while Oromia $(0.5 \%)$ and Tigray $(0.6 \%)$ had lower prevalence [3]. Only $23 \%$ of the households in these regions have access to electricity, $70.1 \%$ have only one room for sleeping, and $52.5 \%$ cook inside the main house, $77.0 \%$ use wood fire as energy source. Regarding education, $50.8 \%$ of women and $29.5 \%$ of men did not attend formal education [27].

\section{Study design}

This is a cross-sectional baseline survey, which is part of a large, longitudinal, multi-site study designed to enrol and monitor cohorts of nets across four regions of Ethiopia over 3 years to assess physical and chemical durability. This study was carried out following the mass LLIN campaign conducted in 2015.

\section{Sample size and sampling procedure}

The sample size for the baseline survey was calculated following the WHO standard for phase III filed trial of nets [11]. The calculation yielded 460 households for each region, by assuming 95\% confidence interval and $80 \%$ power, and a net attrition rate of $20 \%$ per year and $50 \%$ over 3 years. The total sample size for the four regions was 1840 households. Each region constituted a survey domain. A three-stage sampling procedure was used to select households from each region. (1) Districts were defined to belong to low, moderate or high malaria transmission areas. Only districts where the LLINs distribution campaign had already been completed were included in the study. These were identified in consultation with Regional Health Bureau. At the time of the survey, LLIN distribution had taken place in only two and three districts in the low and high transmission areas, respectively. All five districts were included. To select districts from the moderate transmission areas, Excel random generation was used to select seven districts out of 30 eligible districts; (2) Clusters [enumeration areas (EAs) containing 150-200 households] were selected using simple random sampling. To get the required sample size for each region (460) and because 20 households were to be selected for each cluster (see below), a total of 23 clusters were selected across the districts. Clusters were allocated proportionally to the size of the population in each district. On average, eight clusters were selected in each district; and, (3) 20 households were selected from each cluster using systematic random sampling. Data collectors used a household listing and sampling sheets to select the 20 households. All the names of the heads of the households, their receipt of LLINs during the 2015 distribution campaign and their presence at home on 
one of two visit attempts were recording on the sampling sheets. Those households that fulfilled the inclusion criteria were given a sampling number. To get the sampling interval (K), the total number of households included was divided by 20 . To start the sampling, a random number was generated between 1 and $\mathrm{K}$ using a simple lottery method and every Kth household in the sample was visited. The head of household or an adult member of the household (aged 18 years or above) was interviewed.

\section{Data collection procedure}

Data were collected electronically using a structured questionnaire approximately 2 months after LLINs were distributed to households. Questions on household characteristics, socio-demographic factors, knowledge related to net care, exposure to information on net care and repair, and perceptions of net care and repair were asked. The questionnaire was pre-tested in advance of data collection.

Trained data collectors and supervisors conducted data collection using a hand-held tablet device with electronic questionnaire developed using an Open Data Kit (ODK) program [28]. Supervisors reviewed the data and sent them to the server at Addis Continental Institute of Public Health (ACIPH) daily, or as soon as internet connectivity allowed. The data management team at ACIPH downloaded and reviewed the data daily. The team provided feedback to the supervisors in the field in terms of completeness and errors to be fixed.

\section{Measurement}

\section{Socio-demographic variables}

Educational status of the household head was classified as illiterate (person who cannot read and write), able to read and write, primary, secondary, and high school and above. Data on age were collected as a continuous variable and categorized in to groups using 5-year intervals. The economic status of the households was measured based on a composite measure wealth index based on household assets and house condition [29], then categorized into quintiles.

\section{Perception-related variables}

A series of eight Likert-scale statements were presented to the respondent to measure perception towards net care and repair. The responses were captured across a scale of 5 , ranging from completely disagree to completely agree. Additional file 1 shows the eight perception statements used to capture perception towards net care and repair.

The response for each statement was coded as -2 "completely disagree", - 1 "disagree", 0 "neutral", 1 "agree", or 2 "completely agree". To calculate the overall perception score, the response to eight of the perception statements were added-up and divided by 8 to generate mean perception levels for everyone. Based on the mean score, respondents were further categorized as having negative perceptions when their score was $\leq 0$; having positive perception when their score range was between 0.01 and 1.0; and, having very positive perception when their score was between 1.01 and $2.0[19,30]$.

\section{Exposure to information on LLINs}

Participants were asked if they have received information regarding LLINs in the 6 months prior to the survey and their response was coded as yes or no. Participants who said they had received information were then asked what the information was about. From the list of topics, the respondents could provide multiple responses. Participants were also asked if they had discussed net care and repair with their family; their response was captured as "yes" or "no".

\section{Knowledge on net care and repair}

Participants were asked what action they would take to prevent holes. Their response was captured from the following list of actions: "keep away from children"; "keep away from pests and rodents"; "roll-up or tie-up when not in use"; "handle the net with care"; "do not soil with food"; "keep away from flames"; "wash gently"; "wash only when dirty"; "inspect regularly for holes"; "repair small holes quickly". Respondents who stated five or more (e.g., over half) of the correct answers were dichotomized as having knowledge on net care and repair and the remaining as not having knowledge.

\section{Data analysis}

Data analysis was done using STATA version 12 (Stata Corporation, College Station, TX, USA) using the "surveyset" command to account for complex survey data, population weights were also applied to account for unequal probability of selection across some districts. Descriptive statistics across outcomes are provided. Ordinal logistic regression was used to identify factors associated with the perception towards net care and repair. Factors tested include knowledge towards net care and repair, exposure to information on net care and repair, discussion on net care and repair in the family, and number of LLINs owned by the household. The model also controlled for the following socio-demographic variables: age of the respondent, gender, educational status, wealth, and region.

\section{Ethical consideration}

The Institutional Review Board (IRB) at Addis Continental Institute of Public Health (ACIPH) approved the 
protocol. Permission letters were also obtained from each study region (Amhara, Oromia, Tigray, SNNP) and selected districts. At the household level, the study was fully explained to the respondent and a verbal consent was obtained from each participant.

\section{Results}

\section{Socio-demographic characteristics of household} respondent

A total of 1839 households were included in the sample and the response rate was $99.9 \%$; only one house was excluded from the study. The majority of the respondents were male (80.2\%) and head of the household (98.8\%). The mean age of the respondents was 44 years and $24.8 \%$ were 55 years old and above. More than half of the head of the households (53.5\%) reported not attending formal school, suggesting low level of literacy. The average household size was 5.17 persons and ranged from 1 to 12 individuals. More than $90 \%$ of the houses had floors made of earth and $76.5 \%$ of houses used corrugated iron for roofing material (Table 1).

\section{Exposure to information and knowledge on net care and repair}

A quarter of the respondents said they have received information on net care and repair in the 6 months prior to the survey. The most commonly reported topic was "hang-up your net" (72.8\%; 95\% CI 68.6-77.1\%) followed by "care for your net" which was reported by $56.6 \%$ (95\% CI $52.1-61.2 \%$ ) of respondents. Only 3.0\% (95\% CI 1.5$5.1 \%$ ) of the respondents reported receiving information on net repair. Health extension workers (HEWs) were the main source of net care and repair information. Approximately $19 \%$ of respondents reported discussing net care and repair with their family, after being asked about any such discussions. Respondents' knowledge on net care and repair is presented in Table 2.

\section{Perception about net care and repair}

Almost all respondents reported believing that nets are valuable and $96.1 \%$ thought that they could help protect their family from malaria by taking care of their net. Although the majority $(82.4 \%)$ responded that there are ways to make their net last longer, approximately half $(47.2 \%)$ of respondents reported that a repaired net is not effective against mosquito bites. Almost $40 \%$ of respondents thought the repair of nets was not possible, and $32.5 \%$ did not have the confidence to make a repair. Almost a quarter (23.2\%) of respondents indicated insufficient time to repair holes in their net and $47.0 \%$ did not think others in the community repaired holes in their nets either. Overall, $82.4 \%$ of the respondents had either a positive (i.e., perception score was above 0 ) or very
Table 1 Socio-demographic characteristics of households and respondents, Ethiopia 2015

\begin{tabular}{|c|c|c|}
\hline Variables & Percentage (\%) & Standard error \\
\hline \multicolumn{3}{|l|}{ Gender $n=1839$} \\
\hline Male & 80.2 & 0.0096 \\
\hline Female & 19.8 & 0.0096 \\
\hline \multicolumn{3}{|l|}{ Age $n=1839$} \\
\hline $18-29$ & 12.77 & 0.0821 \\
\hline $30-39$ & 30.02 & 0.0113 \\
\hline $40-49$ & 24.50 & 0.0107 \\
\hline $50-59$ & 14.09 & 0.0085 \\
\hline $60+$ & 18.62 & 0.0091 \\
\hline \multicolumn{3}{|l|}{ Mean \pm SD } \\
\hline \multicolumn{3}{|l|}{$44 \pm 0.33$} \\
\hline \multicolumn{3}{|c|}{ Relationship with the household head $n=1839$} \\
\hline Head & 98.8 & 0.0025 \\
\hline Wife of the head & 1.1 & 0.0023 \\
\hline Son/daughter & 0.06 & 0.0006 \\
\hline Grandchild & 0.1 & 0.0008 \\
\hline \multicolumn{3}{|c|}{$\begin{array}{l}\text { Educational status of head of household } \\
n=1839\end{array}$} \\
\hline No formal education & 53.70 & 0.0116 \\
\hline Primary (grade 1-6) & 25.57 & 0.0102 \\
\hline Secondary (grade 7-8) & 8.27 & 0.0064 \\
\hline More than secondary ( $\geq$ grade 9 ) & 12.46 & 0.0077 \\
\hline Mean household size & 5.17 & 0.5126 \\
\hline \multicolumn{3}{|l|}{$\begin{array}{l}\text { Wealth index } \\
\mathrm{n}=1829\end{array}$} \\
\hline 1st & 20.1 & 0.0097 \\
\hline 2nd & 20.0 & 0.0099 \\
\hline $3 r d$ & 20.1 & 0.0099 \\
\hline 4th & 19.9 & 0.0010 \\
\hline 5th & 20.0 & 0.0090 \\
\hline \multicolumn{3}{|l|}{ Region $n=1839$} \\
\hline Tigray & 24.2 & 0.0012 \\
\hline Amhara & 26.4 & 0.0009 \\
\hline Oromia & 27.7 & 0.0016 \\
\hline SNNP & 21.6 & 0.0010 \\
\hline \multicolumn{3}{|l|}{ Household characteristics } \\
\hline \multicolumn{3}{|l|}{ Roofing $n=1839$} \\
\hline Grass/leaf & 19.3 & 0.0073 \\
\hline Mud & 1.1 & 0.0027 \\
\hline Rustic mat/plastic sheets & 2.1 & 0.0033 \\
\hline Corrugated iron/wood & 76.5 & 0.0082 \\
\hline Cement/concrete & 0.7 & 0.0017 \\
\hline Stone & 0.3 & 0.0014 \\
\hline \multicolumn{3}{|l|}{ Floor $n=1839$} \\
\hline Earth & 91.7 & 0.0055 \\
\hline Wood/bamboo/palm & 1.7 & 0.0026 \\
\hline Vinyl/parquet & 0.004 & 0.0004 \\
\hline Tiles/cement & 6.40 & 0.0006 \\
\hline
\end{tabular}

${ }^{a}$ Education categories refer to the highest level of education attended, whether or not that level was completed 
Table 2 Knowledge about net care, repair and exposure to information among household respondents, Ethiopia, 2015

\begin{tabular}{|c|c|c|}
\hline Characteristics & & Percentages $(95 \% \mathrm{Cl})$ \\
\hline \multicolumn{3}{|l|}{ Knowledge about net care and repair $(n=1829)$} \\
\hline Handle the net with care & & $71.4 \%(69.2 \%, 73.5 \%)$ \\
\hline Roll-up or tie-up when not in use & & $63.4 \%(61.3 \%, 65.4 \%)$ \\
\hline Keep away from children & & $47.9 \%(45.5 \%, 50.2 \%)$ \\
\hline Wash gently & & $36.2 \%(33.9 \%, 38.5 \%)$ \\
\hline Keep away from flame/fire & & $35.0 \%(32.8 \%, 37.2 \%)$ \\
\hline Keep away from pests and rodents & & $34.8 \%(32.7 \%, 40.0 \%)$ \\
\hline Wash only when dirty & & $32.3 \%(30.2 \%, 34.6 \%)$ \\
\hline Do not soil with food & & $19.7 \%(18.0 \%, 21.5 \%)$ \\
\hline Inspect regularly for holes & & $17.7 \%(16.0 \%, 19.5 \%)$ \\
\hline Repair small holes quickly & & $13.1 \%(11.6 \%, 14.7 \%)$ \\
\hline At least five correct answers $(n=1761)$ & & $22.3 \%(20.4 \%, 24.3 \%)$ \\
\hline \multicolumn{3}{|l|}{ Exposure to information on net care and repair $(n=1839)$} \\
\hline Received information in the last 6 months & Yes & $24.6 \%(22.7 \%, 26.5 \%)$ \\
\hline \multicolumn{3}{|l|}{ Content of information exposed to } \\
\hline Hang-up your net $(\mathrm{n}=452)$ & Yes & $72.8 \%(68.6 \%, 77.1 \%)$ \\
\hline Care for your net $(n=452)$ & Yes & $56.6 \%(52.1 \%, 61.2 \%)$ \\
\hline Repair your net $(n=452)$ & Yes & $03.0 \%(01.5 \%, 05.1 \%)$ \\
\hline \multicolumn{3}{|l|}{ Source of information $(n=450)$} \\
\hline HEWs/HDAs/other health workers & & $86.1 \%(82.5 \%, 89.0 \%)$ \\
\hline Other sources (community leaders, radio, family or friend) & & $13.9 \%(11.0 \%, 17.5 \%)$ \\
\hline Discussion on net care and repair with a family $(n=1839)$ & Yes & $19.2 \%(17.5 \%, 21.1 \%)$ \\
\hline
\end{tabular}

positive (i.e., perception score was above 1) perception towards net care and repair (Table 3 ).

Using ordinal logistic regression and overall perception levels as an outcome, the odds of positive perception around net care and repair did not increase in relation to exposure to information on net care and repair in the last 6 months, after controlling for socio-demographic variables. However, those who reported discussing net care and repair with their family were more likely to have a positive perception towards net care and repair (OR 1.47, 95\% CI 1.14-1.89) compared to those who did not discuss net care and repair. Similarly, respondents with knowledge about net care and repair had 58\% higher odds of having positive perception towards net care and repair (OR 1.58, 95\% CI 1.23-2.02). The number of nets in the household did not show a statistically significant association with perception towards net care and repair (Table 4).

\section{Discussion}

In general, knowledge about net care and repair was low, as was those reporting to have received information or discussed net care and repair with their families, despite the recent behavioural change communication (BCC) campaign in the study area. This suggests that targeted BCC campaigns should be improved to focus on providing useful information on how to care for nets, thus increasing not only knowledge but also ability to care for and repair nets. However, most respondents did have positive perception towards net care and repair; respondents with knowledge about net care and repair and those who discussed net care and repair with their family were more likely to have positive perception. BCC campaigns should build on this observation and not only target knowledge and perception, but also provide specific guidance on how best to access resources and skills for the maintenance and repair of nets.

Authors of this study are not aware of any studies that captured an overall knowledge score on net care and repair; however, the three most common net care approaches mentioned in this study were also identified in studies elsewhere [19, 20, 25]. Careful handling of net and keeping nets out of children's reach is a common technique used [25]. Repairing small holes quickly is likely the least cited technique in many places, as was found in this study [19]. While information regarding net care and repair would ideally be given out, this study found very few to have received any information. Results of this study suggest that increasing the amount and quality of information on net care and repair may increase positive perceptions, further reinforcing the idea that BCC could be an important tool; other studies have drawn similar conclusions [31]. 
Table 3 Perceptions towards net care and repair among households, in Ethiopia, 2015

\begin{tabular}{|c|c|}
\hline Variables $(n=1829)$ & Percentage $(95 \% \mathrm{Cl})$ \\
\hline \multicolumn{2}{|c|}{ Mosquito nets are valuable } \\
\hline Agree & $99.9 \%(99.7 \%, 100 \%)$ \\
\hline Neutral & $0.02 \%(0.003 \%, 0.16 \%)$ \\
\hline Disagree & $0.05 \%(0.008 \%, 0.38 \%)$ \\
\hline \multicolumn{2}{|c|}{ There are actions to make my net last long } \\
\hline Agree & $82.4 \%(80.5 \%, 84.2 \%)$ \\
\hline Neutral & $4.43 \%(3.5 \%, 5.6 \%)$ \\
\hline Disagree & $13.2 \%(11.7 \%, 14.8 \%)$ \\
\hline \multicolumn{2}{|c|}{ It is not possible to repair holes in net } \\
\hline Agree & $39.6 \%(37.2 \%, 42.0 \%)$ \\
\hline Neutral & $3.68 \%(2.9 \%, 4.7 \%)$ \\
\hline Disagree & $56.8 \%(54.3 \%, 59.2 \%)$ \\
\hline \multicolumn{2}{|c|}{ A repaired net can still be effective } \\
\hline Agree & $47.5 \%(45.1 \%, 49.8 \%)$ \\
\hline Neutral & $5.27 \%(2.9 \%, 4.7 \%)$ \\
\hline Disagree & $47.2 \%(44.9 \%, 49.6 \%)$ \\
\hline \multicolumn{2}{|c|}{ Other people in this community fix holes in their net } \\
\hline Agree & $25.5 \%(23.5 \%, 27.6 \%)$ \\
\hline Neutral & $27.6 \%(25.6 \%, 29.7 \%)$ \\
\hline Disagree & $47.0 \%(44.7 \%, 49.2 \%)$ \\
\hline \multicolumn{2}{|c|}{ Do not have time to repair holes } \\
\hline Agree & $23.2 \%(21.3 \%, 25.3 \%)$ \\
\hline Neutral & $2.46 \%(1.8 \%, 3.4 \%)$ \\
\hline Disagree & $74.3 \%(72.1 \%, 76.3 \%)$ \\
\hline \multicolumn{2}{|c|}{ I can help protect my family from malaria by taking care of my net } \\
\hline Agree & $96.1 \%(95.0 \%, 97.0 \%)$ \\
\hline Neutral & $0.27 \%(0.09 \%, 0.08 \%)$ \\
\hline Disagree & $3.61 \%(2.8 \%, 4.7 \%)$ \\
\hline \multicolumn{2}{|c|}{ I am confident I can repair holes immediately } \\
\hline Agree & $65.4 \%(63.2 \%, 67.6 \%)$ \\
\hline Neutral & $2.02 \%(1.4 \%, 2.9 \%)$ \\
\hline Disagree & $32.5 \%(30.5 \%, 34.7 \%)$ \\
\hline \multicolumn{2}{|l|}{ Overall perception score } \\
\hline Negative & $17.6 \%(15.9 \%, 19.5 \%)$ \\
\hline Positive & $47.3 \%(44.9 \%, 49.7 \%)$ \\
\hline Very positive & $35.1 \%(32.9 \%, 37.4 \%)$ \\
\hline
\end{tabular}

A considerable proportion of respondents had positive perception towards net care and repair. Slightly higher level of positive perception towards net care was however observed across sub-Saharan Africa [19, 30]. This variation could be explained by socio-demographic characteristics such as gender or wealth [21], or perhaps because of study design. In addition, there was variation in the individual perception statements; while a majority of respondents believe they can take care of their nets and protect their family from malaria, not all respondents believed it was possible to repair a net or did not know how. Moreover, other studies have documented a perception that a torn net is no longer useful [23] and there is simply a preference for a new net once a net is torn [26]; both of these perceptions could be targeted by $\mathrm{BCC} /$ net distribution campaigns. In this context, social norms could also be targeted; many respondents thought that their neighbours did not repair nets and this may have influenced their perceptions. Other studies have found social norms to be an important motivator [21, 25].

\section{Limitations}

In general, it is imperative to interpret the results of this study with some inherent caveats of the study design, such as the cross-sectional nature of the study, which may not allow establishing temporal relationship between perception and the exposure variables. In addition, asking questions about net repair 2 months after net distribution may not be ample time to assess repair practices, as many of the new nets are likely still intact. A third limitation relates to the division of labour within households; it is possible that those responsible for net care and repair might not be the head of households, or the adult resident who answered the questions. Thus, one reason why respondents in this study may have little to say about net care and repair is because the study failed to interview the person responsible for net care and repair. Fourth, as this study measured reported behaviour, and reported behaviour is sometimes subject to social desirability bias, especially given the data were collected shortly after the net distribution campaign where recipients could have been exposed to messages that influence their responses. A fifth limitation relates to whether knowledge and perception actually translate into practice; as this study only measured perceptions and knowledge, and not behaviour, it is possible that other factors are interacting to influence repair practices. Repair skill, selfefficacy, availability of repair resources, social norms, and people's expectations about when another net distribution is likely to occur may also influence repair practices in a community. Lastly, it is unclear the extent to which the BCC campaign employed in 2015 actually addressed net care and repair in great detail; while most BCC campaigns focus on promoting net use, information on specific instructions for repairing or caring for nets is often not provided.

\section{Conclusions}

The overall level of knowledge on net care and repair was low in the study areas and repairing holes in the nets was the least frequently mentioned method of net care approach. In addition, not enough information and technical assistance on net care and repair is reaching the communities. Although the overall positive perception 
Table 4 Factors associated with household's perception towards net care and repair, Ethiopia, 2015

\begin{tabular}{|c|c|c|c|c|c|}
\hline & \multicolumn{3}{|c|}{ Perception towards net care and repair } & \multirow{2}{*}{$\begin{array}{l}\text { Adjusted odds } \\
\text { ratio }(95 \% \mathrm{Cl})\end{array}$} & \multirow[t]{2}{*}{$P$ value } \\
\hline & Negative & Positive & Very positive & & \\
\hline \multicolumn{6}{|l|}{ Knowledge on net care and repair } \\
\hline Not adequate & $273(85.8 \%)$ & $657(80.6 \%)$ & $434(69.8 \%)$ & & \\
\hline Adequate knowledge & $45(14.2 \%)$ & $159(19.4 \%)$ & $188(30.3 \%)$ & $1.58(1.23,2.02)$ & $<0.001$ \\
\hline \multicolumn{6}{|c|}{ Exposure to information on net care and repair } \\
\hline No & 294 (91.5\%) & $751(87.0 \%)$ & $519(80.9 \%)$ & 1.00 & \\
\hline Yes & $27(8.5 \%)$ & $113(13.0 \%)$ & $122(19.1 \%)$ & $1.24(0.92,1.65)$ & 0.16 \\
\hline \multicolumn{6}{|c|}{ Discussion on net care and repair in the family } \\
\hline No & $289(89.7 \%)$ & $712(82.4 \%)$ & $476(74.2 \%)$ & 1.00 & \\
\hline Yes & $33(10.3 \%)$ & $152(17.6 \%)$ & $166(25.9 \%)$ & $1.47(1.14,1.89)$ & $<0.001$ \\
\hline \multicolumn{6}{|l|}{ Number of LLINs owned } \\
\hline One & $119(36.9 \%)$ & $270(31.2 \%)$ & $224(35.0 \%)$ & 1.00 & \\
\hline Two & $126(39.1 \%)$ & $369(42.7 \%)$ & $257(40.1 \%)$ & $1.05(0.83,1.33)$ & 0.70 \\
\hline Three or more & $77(24.0 \%)$ & $225(26.1 \%)$ & $160(24.9 \%)$ & $1.10(0.82,1.49)$ & 0.51 \\
\hline \multicolumn{6}{|l|}{ Gender } \\
\hline Male & $248(77.26 \%)$ & $687(79.79 \%)$ & $526(81.93 \%)$ & 1.00 & \\
\hline Female & $73(22.74 \%)$ & $174(20.21 \%)$ & $116(18.07 \%)$ & $0.72(0.56,0.94)$ & 0.06 \\
\hline \multicolumn{6}{|l|}{ Age (years) } \\
\hline $18-29$ & $45(13.98 \%)$ & $108(12.50 \%)$ & $80(12.46 \%)$ & 1.00 & \\
\hline 30-39 & $89(27.64 \%)$ & $264(30.56 \%)$ & $193(30.06 \%)$ & $0.93(0.67,1.30)$ & 0.68 \\
\hline $40-49$ & $70(21.74 \%)$ & $217(25.12 \%)$ & $162(25.23 \%)$ & $0.91(0.64,1.29)$ & 0.58 \\
\hline $50-59$ & $44(13.66 \%)$ & $113(13.08 \%)$ & $100(15.58 \%)$ & $1.06(0.70,1.58)$ & 0.79 \\
\hline $60+$ & $74(22.98 \%)$ & $162(18.75 \%)$ & $107(16.67 \%)$ & $0.80(0.55,1.18)$ & 0.27 \\
\hline \multicolumn{6}{|l|}{ Educational status of head of household } \\
\hline No formal education & $185(57.63 \%)$ & $457(52.95 \%)$ & $336(52.34 \%)$ & 1.00 & \\
\hline Primary (grade 1-6) & 79 (24.61\%) & $223(25.84 \%)$ & $164(25.55 \%)$ & $0.98(0.76,1.26)$ & \\
\hline Secondary (grade 7-8) & $26(8.10 \%)$ & 75 (8.69\%) & $49(7.63 \%)$ & $1.05(0.72,1.52)$ & \\
\hline More than secondary ( $\geq$ grade 9 ) & $31(9.66 \%)$ & $108(12.51 \%)$ & $93(14.49 \%)$ & $1.08(0.78,1.49)$ & \\
\hline \multicolumn{6}{|l|}{ Wealth index } \\
\hline 1st & $73(23.03 \%)$ & $161(18.74 \%)$ & $128(20.00 \%)$ & 1.00 & \\
\hline 2nd & $61(19.24 \%)$ & $179(20.84 \%)$ & $123(19.22 \%)$ & $0.90(0.67,1.23)$ & 0.52 \\
\hline $3 r d$ & $74(23.34 \%)$ & $185(21.54 \%)$ & $106(16.56 \%)$ & $0.70(0.51,0.96)$ & 0.03 \\
\hline 4 th & $63(19.87 \%)$ & $182(21.19 \%)$ & $118(18.44 \%)$ & $0.81(0.59,1.13)$ & 0.22 \\
\hline 5 th & $46(14.51 \%)$ & $152(17.69 \%)$ & $165(25.78 \%)$ & $1.19(0.85,1.67)$ & 0.30 \\
\hline \multicolumn{6}{|l|}{ Region } \\
\hline Tigray & $41(12.73 \%)$ & $212(24.54 \%)$ & $191(29.75 \%)$ & 1.00 & \\
\hline Amhara & 89 (27.64\%) & $218(25.23 \%)$ & $175(27.26 \%)$ & $0.76(0.58,1.00)$ & 0.05 \\
\hline Oromia & $124(38.51 \%)$ & $257(29.75 \%)$ & 124 (19.31\%) & $0.49(0.37,0.65)$ & 0.00 \\
\hline SNNP & 68 (21.12\%) & 177 (20.49\%) & 152 (23.68\%) & $0.69(0.52,0.92)$ & 0.01 \\
\hline
\end{tabular}

The model was controlled for gender, age, educational status, wealth index, and region

Statistically significant association ( $P$ value $<0.05$ ) are indicated in italics

level towards net care and repair was moderate, it was clear that barriers exist and should be addressed as part of national malaria control programme activities. The National Malaria Control Programme in Ethiopia should focus on providing resources and skills for repairing and caring for nets; in addition, it is imperative that programmes begin working to change social norms so that net owners feel that they are expected to care for and repair their nets. This will serve the dual purpose of increasing the longevity of nets, as well as building a cadre of community members skilled in net care and repair. 


\section{Additional file}

Additional file 1. Statement used to measure overall perception towards net care and repair.

\section{Abbreviations}

ACIPH: Addis Continental Institute of Public Health; AOR: adjusted odds ratio; BCC: behaviour change communication; Cl: confidence interval; EA: enumeration area; IRB: Institutional Review Board; LLIN: long-lasting insecticidal net; MIS: Malaria Indicator Survey; ODK: open data kit; PMI: US President's Malaria Initiative; SNNP: Southern Nations Nationalities People; WHOPES: World Health Organization Pesticide Evaluation Scheme.

\section{Authors' contributions}

AZ was involved in the design and development of the study protocol, fieldwork, data management, and analysis and manuscript preparation. YB was involved in the design and development of the study protocol as well as the manuscript. SI led the design and development of the study protocol and reviewed the manuscript. HN coordinated collaboration of different institutes for the development of the study protocol and involved in the development of the study protocol, oversee the logistics and administration of the field work and participated in the supervision of the field work, reviewed the manuscript. SA and HG contributed to the development of the study protocol, supported the fieldwork, and reviewed the manuscript. MD, SC, HS, MY, AK $A W, Y W$, and GD reviewed the protocol and the manuscript. $L M L$ and GP contributed to the development of the study protocol and reviewed the manuscript. JK contributed in the development of the study protocol and preparation of the manuscript. AW was involved in the design and development of the study protocol, and provided support in data management and analysis for the manuscript. All authors read and approved the final manuscript.

\section{Author details}

${ }^{1}$ Addis Continental Institute of Public Health, Addis Ababa, Ethiopia. ${ }^{2}$ Entomology Branch, Division of Parasitic Diseases and Malaria, Center for Global Health, Centers for Disease Control and Prevention and U.S. President's Malaria Initiative, Atlanta, USA. ${ }^{3}$ Ethiopian Public Health Institute, Addis Ababa, Ethiopia. ${ }^{4}$ President Malaria Initiative (PMI-USAID), Addis Ababa, Ethiopia. ${ }^{5}$ Ethiopian National Malaria Prevention, Control and Elimination Program, Addis Ababa, Ethiopia. ${ }^{6}$ London School of Hygiene and Tropical Medicine, London, UK. ${ }^{7}$ Centers for Disease Control and Prevention, Atlanta, USA. ${ }^{8}$ School of Public Health and Tropical Medicine, Tulane University, New Orleans, USA.

\section{Acknowledgements}

The authors thank experts at ACIPH who supervised the field data collection, the IT team and the data management unit who relentlessly worked to maintain data quality, and thank the communities that participated in the study.

\section{Competing interests}

The authors declare that they have no competing interests.

\section{Availability of data and materials}

All the datasets are available on reasonable request to $\mathrm{ACIPH}$.

\section{Ethics approval and consent to participate}

The study was approved by the Institutional Review Board (IRB) at Addis Continental Institute of Public Health (IRB Identification no. 0029 at ESTA).

\section{Funding}

This work was supported by the USAID, US President's Malaria Initiative (PMI) (Grant Number: AID-663-A-14-00004).

\section{Publisher's Note}

Springer Nature remains neutral with regard to jurisdictional claims in published maps and institutional affiliations.

Received: 4 May 2017 Accepted: 21 September 2017

Published online: 02 October 2017
References

1. Ghebreyesus TA, Deressa W, Witten KH, Getachew A, Seboxa T. Malaria. In: Berhane Y, Haile-Mariam D, Kloos H, editors. Epidemiology and ecology of health and disease in Ethiopia. Addis Ababa: Shama Books; 2006. p. 556-76.

2. WHO. World malaria report, 2014. Geneva: World Health Organization; 2014

3. EHNRI. Ethiopia national malaria indicator survey 2011. 2012.

4. Russell TL, Lwetoijera DW, Maliti D, Chipwaza B, Kihonda J, Charlwood JD, et al. Impact of promoting longer-lasting insecticide treatment of bed nets upon malaria transmission in a rural Tanzanian setting with preexisting high coverage of untreated nets. Malar J. 2010;9:187.

5. Lengeler C. Insecticide-treated bed nets and curtains for preventing malaria. Cochrane Database Syst Rev. 2004;2:CD000363.

6. Deressa W, Yihdego YY, Kebede Z, Batisso E, Tekalegne A, Dagne GA. Effect of combining mosquito repellent and insecticide treated net on malaria prevalence in Southern Ethiopia: a cluster-randomised trial. Parasites Vectors. 2014;7:132.

7. Terlouw DJ, Morgah K, Wolkon A, Dare A, Dorkenoo A, Eliades MJ, et al. Impact of mass distribution of free long-lasting insecticidal nets on childhood malaria morbidity: the Togo National Integrated Child Health Campaign. Malar J. 2010;9:199.

8. FMOH. National malaria strategic plan 2014-2020. Addis Ababa: Federal Ministry of Health; 2014.

9. Sena LD, Deressa WA, Ali AA. Predictors of long-lasting insecticidetreated bed net ownership and utilization: evidence from communitybased cross-sectional comparative study, Southwest Ethiopia. Malar J. 2013;12:406.

10. Anemut A. Distribution and utilization of vector control strategies in a malarious village of Jabi Tehnan District, north-western Ethiopia. Malar J. 2014;13:356

11. WHO. Guideline for laboratory and field-testing of long-lasting insecticidal nets. Geneva: World Health Organization; 2013.

12. Wills AB, Smith SC, Anshebo GY, Graves PM, Endeshaw T, Shargie EB, et al. Physical durability of PermaNet 2.0 long-lasting insecticidal nets over three to 32 months of use in Ethiopia. Malar J. 2013;12:244.

13. Lindblade KA, Dotson E, Hawley WA, Bayoh N, Williamson J, Mount D, et al. Evaluation of long-lasting insecticidal nets after 2 years of household use. Trop Med Int Health. 2005;10:1141-50.

14. Tan KR, Coleman J, Smith B, Hamainza B, Katebe-Sakala C, Kean C, et al. A longitudinal study of the durability of long-lasting insecticidal nets in Zambia. Malar J. 2016;15:106.

15. Kilian A, Byamukama W, Pigeon O, Atieli F, Duchon S, Phan C. Long-term field performance of a polyester-based long-lasting insecticidal mosquito net in rural Uganda. Malar J. 2008;7:49.

16. Tami A, Mubyazi G, Talbert A, Mshinda H, Duchon S, Lengeler C. Evaluation of Olyset ${ }^{\mathrm{TM}}$ insecticide-treated nets distributed seven years previously in Tanzania. Malar J. 2004;3:19.

17. Allan R, O'Reilly L, Gilbos V, Kilian A. An observational study of material durability of three World Health Organization-recommended long-lasting insecticidal nets in Eastern Chad. Am J Trop Med Hyg. 2012;83:407-11.

18. Gnanguenon V, Azondekon R, Oke-Agbo F, Beach R, Akogbeto M. Durability assessment results suggest a serviceable life of two, rather than three, years for the current long-lasting insecticidal (mosquito) net (LLIN) intervention in Benin. BMC Infect Dis. 2014;14:69.

19. Helinski MH, Namara G, Koenker H, Kilian A, Hunter G, Acosta A, et al. Impact of a behaviour change communication programme on net durability in eastern Uganda. Malar J. 2015;14:366.

20. Kilian A, Koenker H, Obi E, Selby RA, Fotheringham M, Lynch M. Field durability of the same type of long-lasting insecticidal net varies between regions in Nigeria due to differences in household behaviour and living conditions. Malar J. 2015;14:123.

21. Loll DK, Berthe S, Faye SL, Wone I, Arnold B, Koenker H, et al. "You need to take care of it like you take care of your soul": perceptions and behaviours related to mosquito net damage, care, and repair in Senegal. Malar J. 2014;13:322.

22. Leonard L, Diop S, Doumbia S, Sadou A, Mihigo J, Koenker H, et al. Net use, care and repair practices following a universal distribution campaign in Mali. Malar J. 2014;13:435 
23. Batisso E, Habte T, Tesfaye G, Getachew D, Tekalegne A, Kilian A, et al. A stitch in time: a cross-sectional survey looking at long lasting insecticidetreated bed net ownership, utilization and attrition in SNNPR, Ethiopia. Malar J. 2012;11:183.

24. Mejía P, Teklehaimanot HD, Tesfaye Y, Teklehaimanot A. Physical condition of Olyset ${ }^{\circledR}$ nets after five years of utilization in rural western Kenya. Malar J. 2013;12:158.

25. Hunter GC, Scandurra L, Acosta A, Koenker H, Obi E, Weber R. “We are supposed to take care of it": a qualitative examination of care and repair behaviour of long-lasting, insecticide-treated nets in Nasarawa State, Nigeria. Malar J. 2014;13:320.

26. Central Statistical Agency. Population and housing census report of Ethiopia—2007. Addis Ababa: Central Statistical Agency; 2007.

27. Central Statistical Agency/Ethiopia, ICF International. Ethiopia demographic and health survey 2011. Addis Ababa: Central Statistical Agency/ Ethiopia and ICF International; 2012. p. 2012.
28. Hartung C, Anokwa Y, Brunette W, Lerer A, Tseng C, Borriello G, editors. Open data kit: tools to build information services for developing regions. In: 4th ACM/IEEE int'l conf information and communication technologies and development. 2010.

29. Rutsein SO, Johnson K. The DHS wealth index. DHS comparative reports No. 6. Calverton: ORC Macro; 2004.

30. Koenker H, Kilian A, Hunter G, Acosta A, Scandurra L, Fagbemi B, et al. Impact of a behaviour change intervention on long-lasting insecticidal net care and repair behaviour and net condition in Nasarawa State, Nigeria. Malar J. 2015;14:18.

31. Fuge TG, Ayanto SY, Gurmamo FL. Assessment of knowledge, attitude and practice about malaria and ITNs utilization among pregnant women in Shashogo District, Southern Ethiopia. Malar J. 2015;14:235.

\section{Submit your next manuscript to BioMed Central and we will help you at every step:}

- We accept pre-submission inquiries

- Our selector tool helps you to find the most relevant journal

- We provide round the clock customer support

- Convenient online submission

- Thorough peer review

- Inclusion in PubMed and all major indexing services

- Maximum visibility for your research

Submit your manuscript at www.biomedcentral.com/submit 